\title{
The “AGEING" Experiment in the Spanish Soyuz Mission to the International Space Station
}

E. de Juan (1), A. Benguría (2), A. Villa (2), L. J. Leandro (2), R. Herranz (2), P. Duque (3), E. Horn (4), F. J. Medina (5), J. van Loon (6) and R. Marco (2)

${ }^{(1)}$ División de Fisiología, Universidad de Alicante, E-03080, Alicante, Spain

(2) Departamento de Bioquímica - I.I. Biomédicas "Alberto Sols" (UAM-CSIC), Madrid, Spain

(3) ESA

(4) Department of Neurophysiology, University of Ulm, Germany

${ }^{(5)}$ Centro de Investigaciones Biológicas (CSIC), Ramiro de Maeztu 9, E-28040, Madrid, Spain

(6) Dutch Experiment Support Center, DESC @ OCB-ACTA-Vrije Universiteit, Amsterdam, The Netherlands 


\section{Abstract}

Human exploration of outer space will eventually take place. In preparation for this endeavour, it is important to establish the nature of the biological response to a prolonged exposure to the space environment. In one of the recent Soyuz Missions to serve the International Space Station (ISS), the Spanish Soyuz mission in October 2003, we exposed four groups of Drosophila male imagoes to microgravity during the almost eleven days of the Cervantes mission to study their motility behaviour. The groups were three of young flies and one of mature flies, In previous space experiments, we have shown that when imagoes are exposed to microgravity they markedly change their behaviour by increasing their motility, especially if subjected to these conditions immediately after hatching. The constraints of the current Soyuz flights made it impossible to study the early post-hatching period. A low temperature cold transport was incorporated as a possible way out of this constraint. It turned out that on top of the space flight effects, the cold treatment by itself, modifies the motility behaviour of the flies. Although the four groups increased their motility, the young flies did it in a much lower extent than the mature flies that had not been exposed to the low temperature during transportation. Nevertheless, the flies flown in the ISS are still more active than the parallel ground controls. As a consequence of the lower motility stimulation in this experiment, a likely consequence of the cold transport step, no effects on the life spans of the flown flies were detected. Together with previous results, this study confirms that high levels of motility behaviour are necessary to produce significant decreases in fly longevity.

\section{Introduction}

Many biological beings are going to be part of the adventure of Exploration/colonization of outer space, if not for other reasons in the life support systems in long-term human bases in near-by planets. On the other hand, it is clear that living systems are well adapted to the different conditions existing on the Earth, including gravity. All living organisms are well adapted to its presence. The case of the plants is paradigmatic, but what about animals?. The size and strength of the different biological materials and components is tuned to the presence of the normal gravity on Earth $[1,2]$. Although no changes are expected in short and medium term exposures to different levels of gravity, it can be foreseen that evolutionary changes may appear in the very long-range. On the other hand, behaviour is a more plastic property of living beings, especially in complex multicellular systems $[3,4]$. Thus, it is not surprising that changes in behaviour is one of the traits more often found to change in environmental conditions like altered gravity.

The International Space Station is the current flight facility available to perform experiments in Space to prepare for the new phase of space exploration and colonization. In this article, we will summarize the AGEING experiment run during the Spanish Soyuz Mission, also known as the "Cervantes" Mission.

\section{The AGEING experiment background. The experiment outline}

Drosophila melanogaster is one of the main model systems in current Biology. Most of what we know about how animal development occurs has been derived from information first obtained in this organism. Even the current high-throughput Biology has one of the more solid antecedents in studies initiated more than a century ago in Drosophila [5]. We took advantage of this fact and conceived our AGEING experiment. The experiment intended to study the effects of the space environment on the behavior of Drosophila melanogaster male imagoes exposed to microgravity during the 11 days Soyuz flight. In previous experiments, we had observed a marked increase in the motility of the flies in microgravity, in particular of recently emerged imagoes, while two weeks old imagoes did not respond as much to this exposure [6]. Thus, for the experiment, it was necessary to video record the flies behaviour in the ISS. Since we could not use the only glovebox that had been previously installed in the ISS, we decided to use the miniaquaria previously developed by Prof. E. Horn and EADS-SP/Friedrichshafen. We could easily adapt the miniaquaria for maintaining Drosophila imagoes used in our AGEING experiment. (Fig.1). Even though the experiment could have been flown in the Cervantes Mission in April, as initially envisioned, the delay of six months simplified the actual preparation and completion of the documentation of the experiment.

The experiment focussed on the confirmation of earlier work by us [6] and to extend the information by incorporating different strains of Drosophila. The three strains were: A) "Gravity altered", a strain of flies selected for an altered response to gravity on the ground by using a maze similar to that used by other groups (7). The selection response was to move in the direction of the gravity vector instead, as the normal escape response, going away from that direction, B) "Long lived", a strain of flies that were selected during many years for late reproduction, C) "Short lived", a strain of flies that were selected during many years for early reproduction. We used four experimental conditions: flies as young (recently hatched) as possibly of the three strains and a group of mature, two weeks old flies, of the $\mathrm{C}$ strain. For each condition, two miniaquaria were used, loaded with Drosophila food and 50 male flies. All together the experiment consisted in eight containers.

The main problem was the difficulty of bringing all the flies to Baikonour and preparing there the experiment. We already knew that older flies do not respond so actively in microgravity as the younger ones. We also tested the capability of Drosophila to survive at relatively lower temperatures, which they did. We concluded that we could 
transport the miniaquaria with the flies prepared in Madrid/Toulouse at $14^{\circ} \mathrm{C}$. We could hand over our experiment the night before launch and be able to expose the not so recently emerged imagoes to microgravity at normal temperature. ESA provided the transport container (Yellow Box).

The actual time line of the experiment was as follows. The eight AGEING containers were inserted in the biology transport container, delivered in Baikonour and launched in the Soyuz 7 flight to the ISS on October 18, 2003 at 11.37am local time (5.57 GTM). After docking, the containers were transferred to the ISS, where they were inserted in the holder and installed in the Aquarius 2 incubator in the Russian Segment at $22.5^{\circ} \mathrm{C}$. On the $21^{\mathrm{st}}, 23^{\mathrm{d}}$ and $25^{\text {th }}$ of October at GTM, the eight AGEING containers were removed in groups of four by Pedro Duque, inserted in a specific video holder and each of the two groups of 4 containers video recorded using the IIS Sony Video Camera during 20 minutes (Figs. 1, 2). As indicated, the procedure was repeated three times. The eight containers remained in the Aquarius incubator until October $27^{\text {th }}$, when at 17.45 GTM they were brought into the Biology Transfer Box and transferred to the Soyuz 6 capsule and returned to the ground with Pedro Duque and the exchanged Expedition Six crew in the ISS (Yuri Malenchenko and $\mathrm{Ed} \mathrm{Lu}$ ).

Landing took place on October 28 at 2.45 GMT and the AGEING containers that should have been brought back at room temperature were mistakenly transferred back immediately after landing to the Yellow Box at $3{ }^{\circ} \mathrm{C}$ to be taken back to Moscow where at Star City they were deliver to the scientific team around 16.09 GMT. After moving into the provisional laboratory set by us there the containers were inspected. The flies were in very bad shape due to this unprogrammed second colder transport step. An almost parallel experiment, using flies prepared a day later and exposed to $14^{\circ} \mathrm{C}$ during a similar period of time as for the first transportation step (Toulouse-Baikonour) was performed. The decision of delaying one day the control experiment allowed us to incorporate an equivalent second cold transport at $3^{\circ} \mathrm{C}$ in the Yellow Box, that came with the samples to the laboratory in Star City (Moscow). These control samples had been video recorded on Ground at exactly the same time as the flight ones, but with one day delay.

The samples were analyzed for their behaviour after recovery in Moscow and during their posterior life spans in Madrid. Locomotor behaviour of Drosophila males was analyzed on digitized video records using the SMART v2.5 colour image video-tracking software (Panlab, Barcelona, Spain). Spontaneous global activity was quantified as surface change rate by measuring the number of pixels that change between two consecutive images in a defined arena of the miniaquaria. Global activity was analyzed during the first 3 minutes of video recording.

\section{Results of the AGEING Experiment}

In Fig. 3a, a representation of the motility of the flies in the Spanish Soyuz Mission in Space is shown. When compared to the parallel controls (Fig. 3b) which have undergone the same treatment but the Space flight, it turned out that a relatively large proportion of them showed higher motility levels. On the other hand, the level of motility was greater in this experiment in the case of the mature flies than in the other groups. In the Fig. 3c we show the level of motility of normal young flies in an earlier experiment video recorded in the Biorack glovebox flown in the Shuttle (IML-2 mission). Evidently, the motility of the mature flies was lower than the young IML-2 male flies. The main difference was that the mature flies in the Spanish Soyuz Mission were not exposed to a lower temperature transport step while the young flies in this experiment were. Two additional features of the experiment are in agreement with these results, namely, the posterior life spans and mortality curves (not shown). In previous exposures to the Space Environment (for example, Benguria et al, 1996), a significant decrease in the life-span of the young male imagoes that had experienced a marked increase in motility had been found. The absence of such an effect in the experiment of the Cervantes mission likely induced by the cold transportation step, did not produce any modifications in the life spans of the different populations of flies. On the other hand, the unprogrammed cold step introduced during the transport from the landing site to Baikonour had an effect in the reduced survival of the populations exposed to the Space environment, not reproduced in the ground control populations.

We analyzed how many of the genes affected in Space are modified in microgravity (Herranz et al., this volume) and to similar stressful conditions (Marco et al, in the press). Finally, we are carrying a similar comparison with the genes modified in ICE First, the Caenorhabditis elegans experiment run in the DELTA mission (Leandro et al, in preparation). The connection between the molecular effects at the gene level and phenotypic traits such as motility behaviour or the ageing response of organisms is still a problem that remains to be tackle by biological science.

\section{The continuation of the analysis}

Performing space flight studies imposes various constraints to the experiment setup. Until a new flight opportunity becomes available to us, we are limited to perform experiments in the ground simulation facilities available in our laboratories. So far, we are performing similar experiments in the Random Position Machine (8), with and without a cold transport step. Magnetic levitation provides a different approach on the ground to simulate microgravity (9). Analyses of experiments equivalent to the AGEING protocol using these ground instrumentation are now in progress. In particular, we are intrigued by the potentiation/neutralization of the phenotypic 
changes by introducing additional modifications in the growing conditions, such as temperature, weak mutations that by themselves may not give sufficient phenotypes, etc.

\section{Acknowledgements}

The skillful work of the crew of the Cervantes mission, P. D., Alexander Kaleri and Michel Foale, in the operation of the procedures in flight on board of the ISS is gratefully acknowledged. We also appreciate the support of the ESA team, in particular Mr Jesús Jiménez, Ms Nathalie Pottier and Mr. Fabrizio Festa. This work was supported by Grants from the Spanish "Plan Nacional de Investigación Científica y Desarrollo Tecnológico" Ref. Nos. ESP2001-4522-PE, and ESP2003-09475-C02-01 (RM), from the Dutch Space Research Organisation, SRON, MG-057 (JvL) and from the German Space Agency DLR, grant 50WB0140 (EH).

\section{References}

1 Vogel, S. Living in a physical world VII. Gravity and life on the ground. J. Biosci. 31(2), 201-214 (2006).

2 Marco R, Husson D, Herranz R, Mateos J, Medina FJ. Drosophila melanogaster and the future of 'evo-devo' biology in space. Challenges and problems in the path of an eventual colonization project outside the earth. . Adv Space Biol Med.; 9:41-81 (2003)

3 Horn, E. R. The development of gravity sensory systems during periods of altered gravity dependent sensory input. Adv Space Biol Med. 9:133-71 (2003)

4 Slenzka, K. Neuroplasticity changes during space flight. Adv Space Res. 31(6):1595-604 (2003)

5 Beller M., Oliver B. One hundred years of high-throughput Drosophila research. Chromosome Res. 14 (4): 349-62. (2006)

6 Benguria, A.; Grande, E.; de Juan, E.; Ugalde, C.; Miquel, J.; Garesse, R.; Marco, R. Microgravity effects on Drosophila melanogaster behavior and aging. Implications of the IML-2 experiment. J. Biotechnol. 47(2-3): 191-201 (1996)

7 Armstrong, J. D., Texada, M. J., Munjaal, R., Baker, D. A., Beckingham, $K . \quad M$. Gravitaxis in Drosophila melanogaster: A genetic screen. Genes, Brain and Behaviour 5, 222-239 (2006).

8 Kraft TF, van Loon JJ \& Kiss JZ. Plastid position in Arabidopsis columella cells is similar in microgravity and on a random-positioning machine. Planta. 211(3):41522(2000).

9 Berry, $M V$ and Geim, A K, Of flying frogs and levitrons' Eur.J.Phys 18, 307-313(1997). 

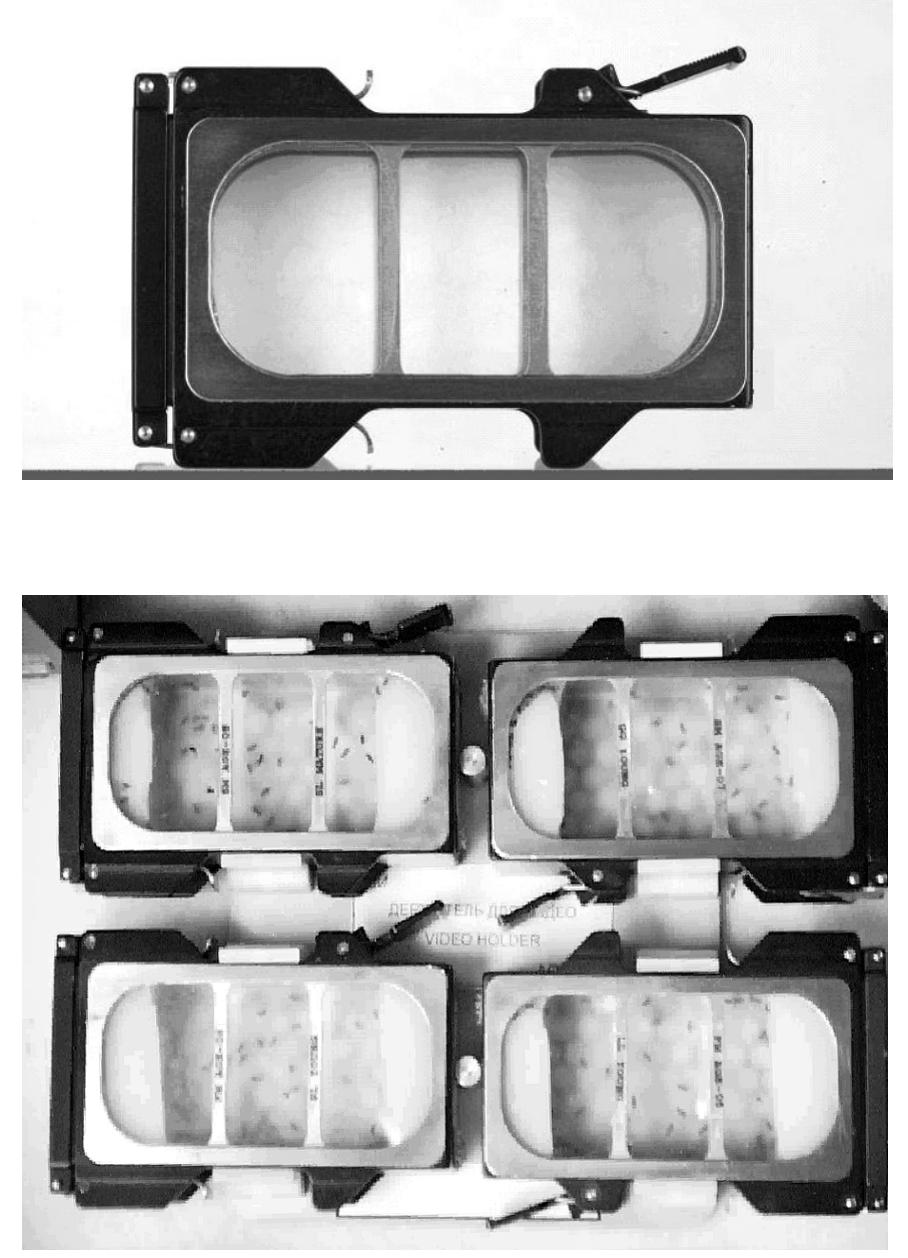

Fig. 1. Miniaquaria with flies (lower frame). Image from the flight video recording. Above a larger view of the container. Dimensions of the type I container: $9.0 \times 4.0 \times 2.5 \mathrm{~cm}$.

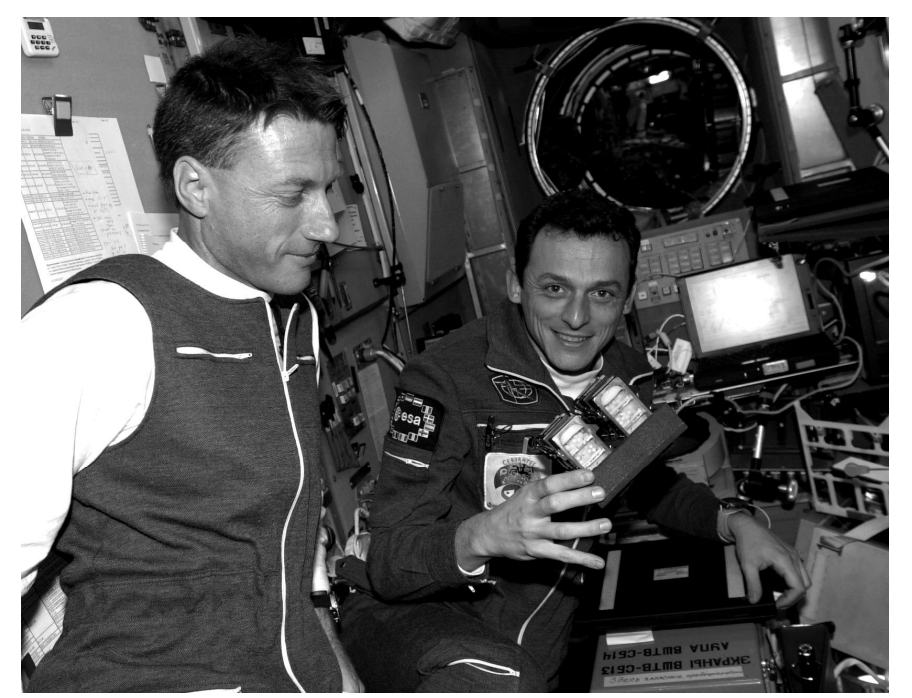

Fig. 2. Extracting the miniaquaria from the ISS incubator. Mike Foal (left) and Pedro Duque (right). 
(a)

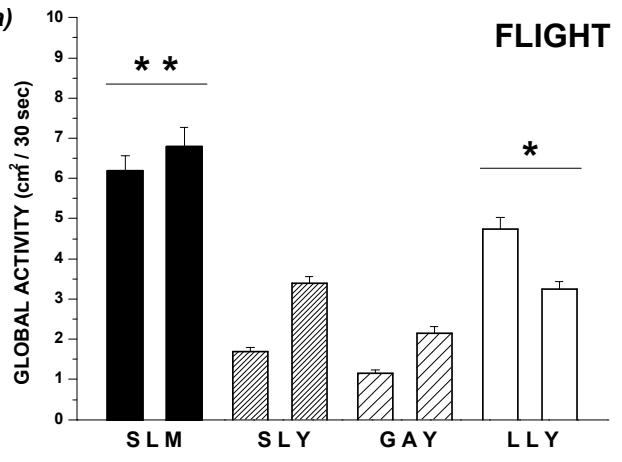

(c, d) IML-2 Mission

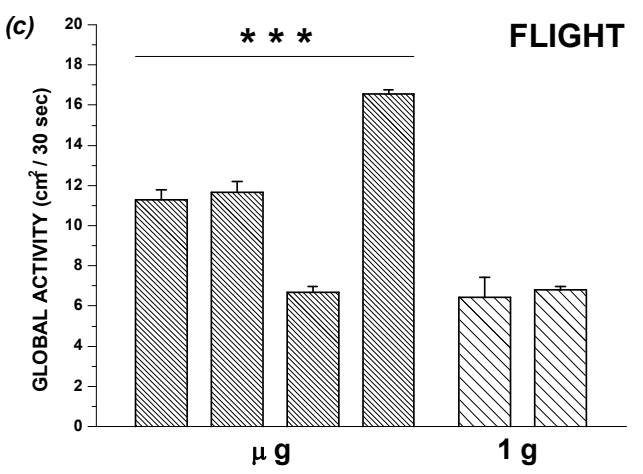

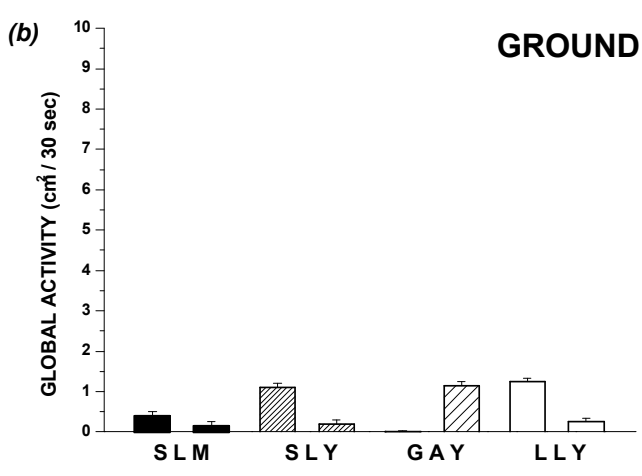

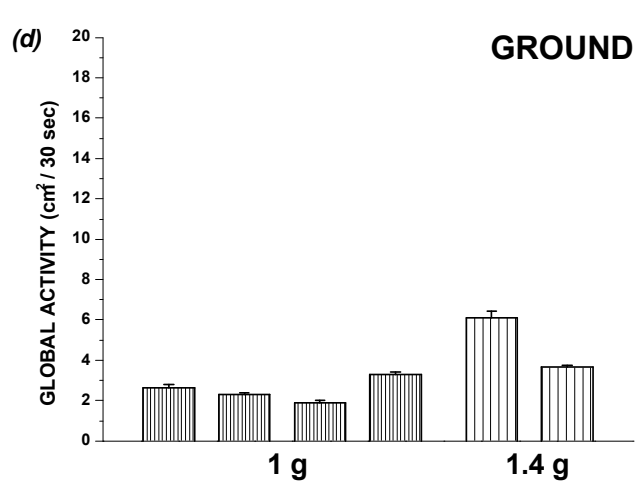

Fig.3. Averaged locomotor activity measured during three minutes of video recording in the Cervantes Soyuz Mission and in the IML-2 Mission. (a) Cervantes Flight activity of different strains in miniaquiaria (two containers per strain): short lived mature flies (SLM), short lived young flies (SLY), gravity altered young (GAY) and long lived young (LLY). (b) Cervantes Ground control activity. The analysis corresponds to the recording on day $6^{\text {th }}$ of the mission. (c) IML-2 Flight activity: young male flies exposed to microgravity ( $\mu$ g) and young male flies installed in the $1 \mathrm{x}$ g Space control centrifuge (1g). (d) IML-2 Ground control activity: young male flies maintained in ground control conditions $(1 \mathrm{~g})$ and young male flies maintained in ground centrifuge conditions $(1.4 \mathrm{~g})$. Recordings also correspond to the end of day $5^{\text {th }}$ of the mission. Note the different scale between the Cervantes and IML-2 experiments. Bars correspond to standard error of the mean. The activity of the SLM flies is significatively different from the activity recorded in the rest of the Cervantes containers $(* *$ p $<0.01$; Bonferroni's Anova multiple comparisons test). The flight containers with young flies exposed to the cold transportation had higher activity than the corresponding ground controls. In particular, the differences shown by the LLY strain are statistically significant $(* p<0.05)$. The activity in the $\mu g$ IML-2 containers is also significatively different from the activity in the rest of the containers $(* * * \mathrm{p}<0.001)$. The activity in the $1 \mathrm{~g}$ flight containers and the activity in the $1.4 \mathrm{~g}$ ground containers coming from the centrifuge is not significatively different, even though the first group had been video recorded at $\mu \mathrm{g}$ and the second at $1 \mathrm{~g}$. 